\section{(2) \\ BRAZIILIAN JOURNAL \\ OF MEDICAL AND BIOLOGICAL RESEARCH}

www.bjournal.com.br
ISSN 0100-879X

Volume 45 (5) 376-472 May 2012

BIOMEDICAL SCIENCES

AND

CLINICAL INVESTIGATION

Braz J Med Biol Res, May 2012, Volume 45(5) 408-410

doi: $10.1590 / \mathrm{S} 0100-879 X 2012007500023$

Restoration of the rabbit corneal surface after total epithelial debridement and complete limbal excision

S.J. Faria-e-Sousa and A. Haddad

The Brazilian Journal of Medical and Biological Research is partially financed by

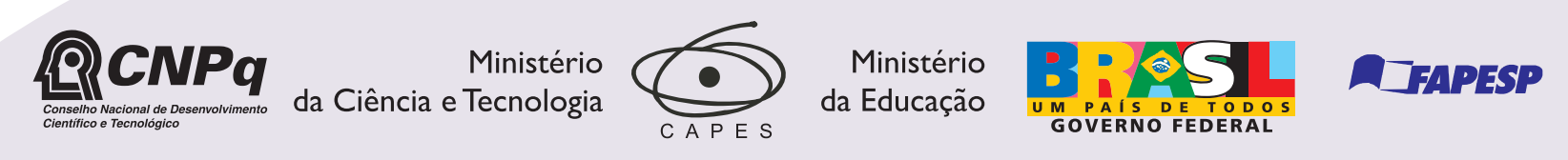

Institutional Sponsors

sompos
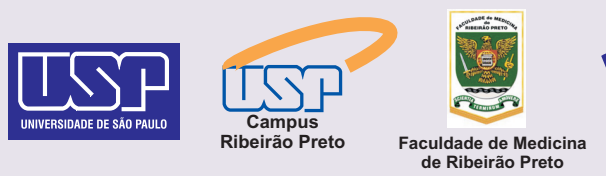

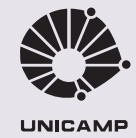

UNICAMP $\oplus$ SHIMADZU

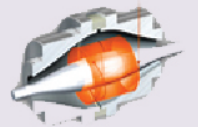

1DI Associaçăo Explore High - Performance MS Orbitrap Technology In Proteomics \& Metabolomics analitica $\underset{\text { analiticaweb.com.br }}{\text { Thermo }}$ 


\title{
Restoration of the rabbit corneal surface after total epithelial debridement and complete limbal excision
}

\author{
S.J. Faria-e-Sousa ${ }^{1}$ and A. Haddad ${ }^{2}$ \\ ${ }^{1}$ Departamento de Oftalmologia, Faculdade de Medicina de Ribeirão Preto, \\ Universidade de São Paulo, Ribeirão Preto, SP, Brasil \\ 2Departamento de Biologia Celular, Faculdade de Medicina de Ribeirão Preto, \\ Universidade de São Paulo, Ribeirão Preto, SP, Brasil
}

\begin{abstract}
How is the corneal epithelium restored when all of it plus the limbus have been eliminated? This investigation explored the possibility that this may be achieved through the conjunctival epithelium. The corneal epithelium of the right eye of 12 rabbits (Oryctolagus cuniculus) was totally scraped followed by surgical excision of the limbus plus 1.0-1.5 mm of the adjacent conjunctiva. Antibiotics and corticosteroids were applied for 1 week after surgery. Histological and immunohistochemical techniques were used to monitor the events taking place on the eye surface 2 weeks and 1, 3 and 6 months thereafter. Initially, the corneal surface was covered by conjunctival-like epithelium. After 1 month and more prominently at 3 and 6 months an epithelium displaying the morphological features of the cornea and reacting with the AE5 antibody was covering the central region. It is likely that the corneal epithelium originated from undifferentiated cells of the conjunctiva interacting with the corneal stroma.
\end{abstract}

Key words: Corneal epithelium; Conjunctiva; Stem cells; Epithelium transformation

\section{Introduction}

The foremost surface of the eye is covered by the corneal epithelium, which exhibits unique features concerning refractive properties, innervation, transparency, and relationship with an avascular and transparent stroma $(1,2)$. Its capability for self-renewal under physiological conditions (3), as well as after injuries by partial scraping $(4,5)$, has been demonstrated. Total loss of the corneal epithelium either follows accidental events or is intentionally achieved by surgical procedures (6). When this occurs, the eye surface becomes sheltered by an epithelium that originates from the proliferation and sliding of the conjunctival epithelium (7). This newly formed epithelium does not replicate the original corneal covering since its refractive and protective properties do not match those of the original corneal epithelium. Furthermore, the stroma becomes vascularized particularly at the periphery. These changes impair proper vision because most of the refractive events take place on the corneal surface (7). Therefore, in order to reinstate good vision it may be assumed that eventually a reaction will occur to restore the corneal surface. The main question is: how is the corneal epithelium restored when all of it plus the limbus have been eliminated?

The purpose of this investigation was to monitor by histological and immunohistochemical techniques the events on the rabbit eye surface after total debridement of the corneal epithelium plus surgical excision of the limbus.

\section{Material and Methods}

Twelve male albino rabbits (Oryctolagus cuniculus) were anesthetized with an intramuscular injection of ketamine hydrochloride (50 mg/kg, Ketamine, Parke Davis, USA) and xylazine hydrochloride (4 mg/kg; Coopazine, Schering Plough Coopers, Brazil). Additional topical anesthesia was provided with proparacaine hydrochloride eye drops (Allergan Inc., USA). The corneal epithelium of the right eye of each rabbit was totally scraped with an ophthalmic spatula, followed by surgical excision of the limbus plus 1.0-1.5 $\mathrm{mm}$ of the adjacent conjunctiva. The excised tissue was processed for histological examination. The eyes

Correspondence: S.J. Faria-e-Sousa, Rua Ottorino Rizzi, 694, 14110-000 Ribeirão Preto, SP, Brasil. Fax: +55-16-3623-2956.

E-mail: sidneyjfs@gmail.com

Received October 4, 2011. Accepted February 16, 2012. Available online March 2, 2012. Published May 7, 2012. 
were treated for one week with eye drops containing ofloxacin and dexamethasone.

At 2 weeks and 1, 3, and 6 months after surgery 3 rabbits per time interval were killed by a lethal intravenous dose of sodium thiopental (Thiopentax, Cristália Produtos Farmaceuticos Ltda., Brazil). The eyes were enucleated and fixed in $4 \%$ formaldehyde. The anterior segment was isolated and processed for paraffin embedding with the exclusion of the lens. Sections were stained with the periodic acid-Schiff (PAS) plus hematoxylin for morphological studies. Other sections were immunologically stained using the primary unlabeled AE5 antibody (Santa Cruz Biotechnology, USA) for the detection of keratins 3 and 12 (8), followed by the goat anti-mouse $\mathrm{F}(\mathrm{ab})$ fragment labeled with horseradish peroxidase (Sigma Aldrich, USA). The reaction was monitored by treating the microscope slides with 3,3'diaminobenzidine plus hydrogen peroxide. Other sections were treated equally, omitting the primary antibody and were used as a control for the immunohistochemical reaction.

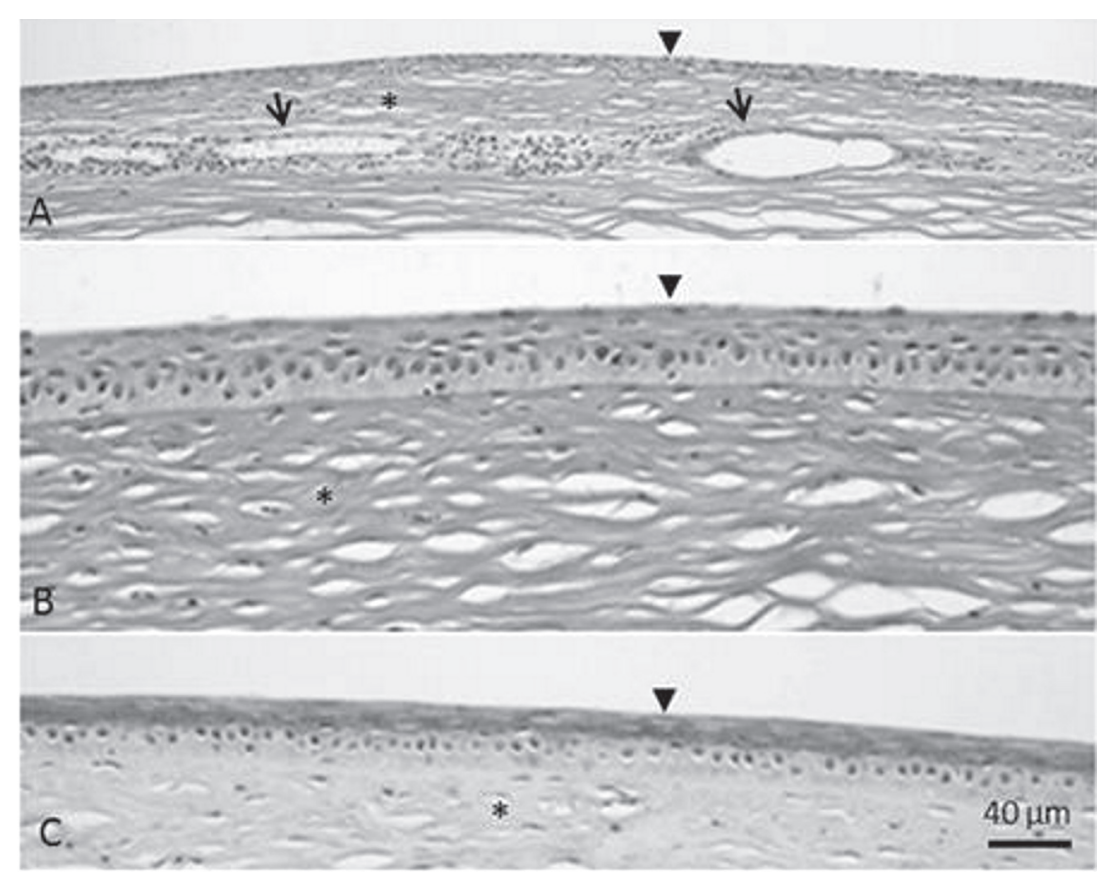

Figure 1. Light microscopy of a rabbit cornea 3 months after surgical excision of the limbus plus $1.0-1.5 \mathrm{~mm}$ of the adjacent conjunctiva. $A$, The peripheral region of the cornea exhibits an epithelium (arrowhead) with conjunctival features and a stroma (asterisk) with many blood vessels (arrows). Periodic acid Schiff (PAS) and hematoxylin staining. $B$, Photomicrograph taken at $3.5 \mathrm{~mm}$ from the border of the Descemet membrane. The epithelium is stratified with morphological features of the corneal epithelium (arrowhead). The basal cells are negatively stained with PAS whereas the suprabasal ones are positive. C, Immunological stain for the detection of keratins 3 and 12 (AE5 antibody). A positive reaction is observed only in the suprabasal cells (arrowhead). The basal stratum is negatively stained. The asterisk indicates the stroma.

\section{Results}

antibody was detected only in the suprabasal layers of this newly formed epithelium, a feature expected to be found only in the limbus of normal rabbits (Figure 1C). At the corneal periphery (1-3 mm from the border of the Descemet membrane) the epithelium exhibited conjunctival features (Figure $1 \mathrm{~A})$ and was negative for the primary AE5 antibody.

\section{Discussion}

It is likely that the conjunctival epithelium, while sliding onto the corneal stroma, carried undifferentiated basal cells, and that these cells, interacting with the corneal stroma, became corneal epithelium cells. This change could have been stimulated by p63, a factor known to promote epithelial stratification and to be prominent in epithelia under regeneration $(9,10)$. The corneal phenotype was confirmed by the reaction of the newly formed epithelium with the AE5 antibody. This antibody reacts with keratins 3 and 12, which are known to occur only in the corneal epithelium, being absent in the conjunctiva. The lack of staining of central basal cells by the AE5 antibody suggests their intermediate stage of differentiation towards regular basal cells. It is 
remarkable that corneal epithelium re-appeared in the center and not at the periphery of the cornea where the stroma remained vascularized for the duration of the experiment. Signs of inflammation such as leukocyte infiltration were conspicuous at the corneal periphery where no epithelium with a corneal phenotype could be seen at any time.

Antibiotics and corticosteroids were employed for only 1 week after surgery. Yet, the epithelial changes occurred much later. Since the limbus was thoroughly excised it may be inferred that the presence of the corneal epithelium was a natural reaction of the eye with no influence of the socalled limbal stem cells. Much more likely is the existence of oligopotent germinative cells covering the anterior surface of the eye that express different phenotypes according to the stromal environment (11). Interaction between epithelium and stroma is a basic tenet, occurring either during embryonic life or after birth, as well as in the course of eye development (1). Therefore, it is highly probable that the interactions between an apparently normal stroma and

\section{References}

1. Bron AJ, Tripathi RC, Tripathi BJ. Wolff's anatomy of the eye and orbit. 8th edn. London: Chapman \& Hall Medical; 1997.

2. Busacca A. Anatomie de la cornée. Vol 1. In: Anonymous, Biomicroscopie et Hitopathologie de L'Oeil. Zurich: Shweizer Druck Und Verlagshaus; 1952. p 179-194.

3. Haddad A. Renewal of the rabbit corneal epithelium as investigated by autoradiography after intravitreal injection of 3H-thymidine. Cornea 2000; 19: 378-383.

4. Barbosa FL, Goes RM, de Faria-E-Sousa SJ, Haddad A. Regeneration of the corneal epithelium after debridement of its central region: an autoradiographic study on rabbits. Curr Eye Res 2009; 34: 636-645.

5. de Faria-E-Sousa SJ, Barbosa FL, Haddad A. Autoradiographic study on the regenerative capability of the epithelium lining the center of the cornea after multiple debridements of its peripheral region. Graefes Arch Clin Exp Ophthalmol 2010; 248: 1137-1144.

6. Kruse FE, Chen JJ, Tsai RJ, Tseng SC. Conjunctival trans- conjunctival epithelium in the center of the cornea caused the transformation of undifferentiated cells of the sliding conjunctival epithelium into basal cells of the corneal epithelium. The lack of corneal epithelium phenotype at the periphery of the cornea could be explained by the negative influence of the inflammation on the stromal microenvironment, preventing the correct interactions needed for appropriate epithelial differentiation.

If the events taking place in the center of the cornea could be understood and controlled, a realistic perspective could be opened to obtain healing of lesions of the eye surface.

\section{Acknowledgments}

The authors acknowledge Vani M. Alves, Domingos S. Souza Filho and José A. Maulin for their technical assistance. Research supported by CNPq, FAPESP, and FAEPA-HC-FMRP. differentiation is due to the incomplete removal of limbal basal epithelium. Invest Ophthalmol Vis Sci 1990; 31: 19031913.

7. Huang AJ, Tseng SC. Corneal epithelial wound healing in the absence of limbal epithelium. Invest Ophthalmol Vis Sci 1991; 32: 96-105.

8. Schermer A, Galvin S, Sun TT. Differentiation-related expression of a major $64 \mathrm{~K}$ corneal keratin in vivo and in culture suggests limbal location of corneal epithelial stem cells. $J$ Cell Biol 1986; 103: 49-62.

9. Blanpain C, Fuchs E. p63: revving up epithelial stem-cell potential. Nat Cell Biol 2007; 9: 731-733.

10. Chahud F, Ramalho LN, Ramalho FS, Haddad A, RoqueBarreira MC. The lectin $\mathrm{KM}+$ induces corneal epithelial wound healing in rabbits. Int J Exp Pathol 2009; 90: 166173.

11. Majo F, Rochat A, Nicolas M, Jaoude GA, Barrandon $Y$. Oligopotent stem cells are distributed throughout the mammalian ocular surface. Nature 2008; 456: 250-254. 